\title{
Conditional control of donor nuclear spins in silicon using Stark shifts
}

\author{
Gary Wolfowicz, ${ }^{1,2, *}$ Matias Urdampilleta, ${ }^{1}$ Mike L. W. Thewalt, ${ }^{3}$ Helge Riemann, ${ }^{4}$ \\ Nikolai V. Abrosimov, ${ }^{4}$ Peter Becker, ${ }^{5}$ Hans-Joachim Pohl, ${ }^{6}$ and John J. L. Morton ${ }^{1,7},{ }^{\dagger}$ \\ ${ }^{1}$ London Centre for Nanotechnology, University College London, London WC1H 0AH, UK \\ ${ }^{2}$ Dept. of Materials, Oxford University, Oxford OX1 3PH, UK \\ ${ }^{3}$ Dept. of Physics, Simon Fraser University, Burnaby, British Columbia V5A 1S6, Canada \\ ${ }^{4}$ Institute for Crystal Growth, Max-Born Strasse 2, D-12489 Berlin, Germany \\ ${ }^{5}$ Physikalisch-Technische Bundesanstalt, D-38116 Braunschweig, Germany \\ ${ }^{6}$ Vitcon Projectconsult GmbH, 07745 Jena, Germany \\ ${ }^{7}$ Dept. of Electronic 83 Electrical Engineering, University College London, London WC1E $7 J E$, UK
}

(Dated: July 19, 2021)

\begin{abstract}
Electric fields can be used to tune donor spins in silicon using the Stark shift, whereby the donor electron wave function is displaced by an electric field, modifying the hyperfine coupling between the electron spin and the donor nuclear spin. We present a technique based on dynamic decoupling of the electron spin to accurately determine the Stark shift, and illustrate this using antimony donors in isotopically purified silicon-28. We then demonstrate two different methods to use a DC electric field combined with an applied resonant radio-frequency (RF) field to conditionally control donor nuclear spins. The first method combines an electric-field induced conditional phase gate with standard RF pulses, and the second one simply detunes the spins off-resonance. Finally, we consider different strategies to reduce the effect of electric field inhomogeneities and obtain above $90 \%$ process fidelities.
\end{abstract}

The manipulation of donor spins in silicon is mainly realised by the application of resonant AC magnetic fields. These can be used to globally control a large ensemble of spins with high fidelity [1, and also to control a single spin [2. However, applying local AC magnetic fields to donors in an interacting array is practically challenging, especially considering the number of high frequency, high power microwave lines in a mature device. For such reasons, the majority of scalable quantum computing architectures based on donors in silicon [3 5], combine globally applied AC magnetic fields with the ability to electrically tune the donor spin, via the Stark effect which primarily modulates the hyperfine interaction between the electron and nuclear spin.

The Stark effect arises from a perturbation of the electron wave function as it is pulled away from the nucleus, mixing its ground state energy level with the higher orbital excited states [6]. Following the original proposal by Kane [3], several theoretical studies have examined Stark-tuning of both donor spins [6], while experiments have focused on measuring the Stark shift parameters using electron spin resonance (ESR) of the donor [1012. These measurements showed a typical Stark-shift induced change in the hyperfine coupling on the order of $\mathrm{kHz}$ (for electric fields around $0.1 \mathrm{~V} / \mu \mathrm{m}$ ). This falls well within the ESR linewidth $(12 \mathrm{MHz}$ in natural silicon, or typically $100 \mathrm{kHz}$ in silicon-28 limited by the magnetic field homogeneity), making it impossible to Stark-shift the electron spin by more than a linewidth.

The nuclear spin, on the other hand, typically has much smaller spin resonance linewidth compared to the electron spin $\left(\gamma_{\mathrm{n}} / \gamma_{\mathrm{e}}=\sim 4 \times 10^{-4}\right.$, where $\gamma_{\mathrm{n} / \mathrm{e}}$ is the gyromagnetic ratio of the nuclear/electron spin) and yet the effect of the Stark-tuned hyperfine shift on the nuclear spin resonance frequency is nearly the same as for the electron spin. As a consequence, using the Stark effect to tune spins in and out of resonance with globally applied fields becomes more readily achievable. Furthermore, the coherence times of the nuclear spin can reach minutes in ensembles [13] and seconds even in single spin devices [14, allowing a much larger number of Starkshift-controlled quantum operations to be applied. In this Letter, we examine the use of the Stark shift to conditionally manipulate the nuclear spin of antimony donors in silicon. We further demonstrate how the Stark shift can either be used as the basis for a simple controlledphase gate or as a detuning gate to enable or disable the effect of a resonant magnetic radio frequency ( $\mathrm{RF}$ ) pulse. In the latter case, we are able to reach process fidelities above $90 \%$ fidelities.

Measurements were conducted using an isotopically enriched silicon-28 float-zone crystal doped with antimony $\left({ }^{121} \mathrm{Sb}\right)$ at a concentration of $10^{14} \mathrm{~cm}^{-3}$. Pulsed electron spin resonance (ESR) and electron-nuclear double resonance (ENDOR) experiments were realised in a Bruker $\mathrm{X}$-band $(\approx 0.3 \mathrm{~T}, 9.7 \mathrm{GHz})$ Elexsys system. The sample, $1.71 \mathrm{~mm}$ in thickness, was inserted between two metal plates connected to a semi-rigid copper nickel coaxial cable to apply the voltage pulses (see Supplementary Information), and sat in a continuous-flow helium cryostat at around $4.5 \mathrm{~K}$. Voltage pulses up to $150 \mathrm{~V}$, equivalent to $0.09 \mathrm{~V} / \mu \mathrm{m}$, were created with a low power high-voltage amplifier. The electric field was applied parallel to the static magnetic field.

In order to measure the amplitude and distribution of the Stark shift in our system, we first confirm and then 
(a)
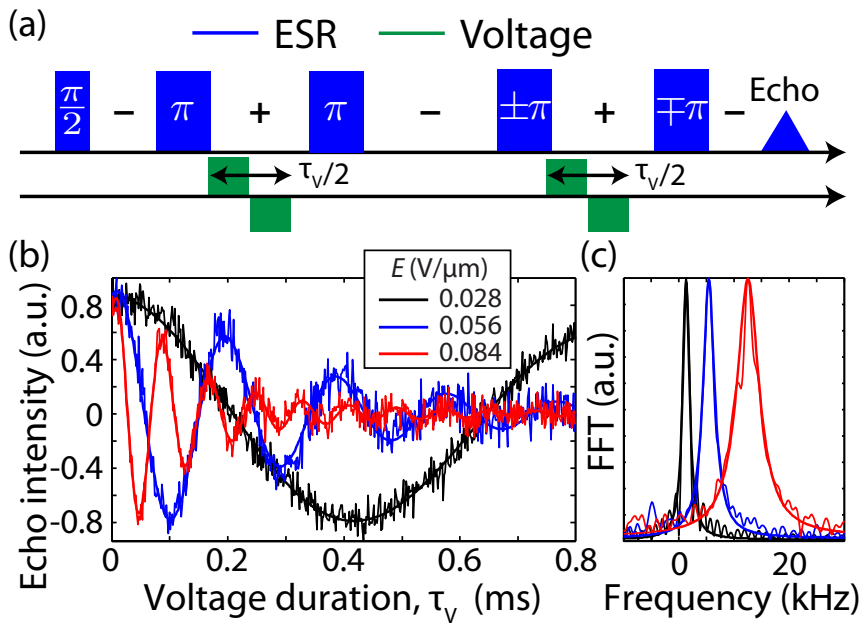

FIG. 1. Measurement of the Stark shift in ${ }^{28} \mathrm{Si}^{121} \mathrm{Sb}$ using dynamical decoupling. (a) Uhrig Dynamical Decoupling (UDD) sequence with four refocusing pulses. As each $\pi$ pulse reverses the phase acquisition (see signs in sequence), the DC electric field is applied in between alternating pairs of $\pi$ pulses. Using bipolar (positive and negative) voltage pulses, the linear Stark shift contribution is eliminated and only the quadratic part remains. The last two ESR pulses were phase cycled to remove any stimulated echoes. (b) Electron spin phase evolution measuring using the $m_{\mathrm{I}}=-5 / 2$ ESR transition for different electric fields. (c) Fourier-transform showing the frequency shift distribution in the sample with a Lorentzian fit.

extend the measurements by Bradbury et. al. [10, on the $\mathrm{Sb}$ donor electron spins. The Stark shift is measured as the electric sensitivity $\eta_{\mathrm{A}}$ and $\eta_{\mathrm{ge}}$ of the hyperfine contact interaction $A$ and the electron g-factor $g_{\mathrm{e}}$, respectively $\left(\left|\gamma_{\mathrm{e}}\right|=g \mu_{\mathrm{B}}\right.$, with $\mu_{\mathrm{B}}$ the Bohr magneton). Together, the application of an electric field $E$ results in a total frequency shift, for a particular electron or nuclear spin transition:

$$
\Delta f(E)=\left[\left(\eta_{\mathrm{A}} A\right) \times \frac{d f}{d A}+\left(\eta_{\mathrm{g}_{\mathrm{e}}} g_{\mathrm{e}}\right) \times \frac{d f}{d g_{\mathrm{e}}}\right] E^{2}
$$

The derivatives $\frac{d f}{d A}$ and $\frac{d f}{d g_{\mathrm{e}}}$ are dependent on the static magnetic field, $B_{0}$, due to possible mixing between the electron and nuclear states, especially at low fields. In the high field limit, for an electron spin transition, $d f / d A$ is equal to $m_{\mathrm{I}}$ (the nuclear spin projection) and $\frac{d f}{d g_{\mathrm{e}}}$ is equal to $\mu_{\mathrm{B}} B_{0}$, while for a nuclear spin transition, $d f / d A$ is equal to $1 / 2$ and $\frac{d f}{d g_{\mathrm{e}}}$ is 0 .

The frequency response is quadratic in the electric field, owing to the tetrahedral symmetry at each donor sites. However, any perturbation from this highsymmetry site (e.g. arising from local strains or internal electric fields from trapped charges in the crystal) gives an additional, linear Stark shift component. The effect of this linear term can be suppressed by the application of alternating positive and negative voltage pulses as introduced by Bradbury et al. [10] and used here.
The Stark shift is measured in a Ramsey-type ESR experiment where the frequency shift $\Delta f(E)$ is acquired as a phase shift in the electron spin over time. In addition, refocusing pulses, such as used in a Hahn echo sequence, can significantly extend the acquisition time. The $T_{2}$ of the electron spin is $7 \mathrm{~ms}$, limited by instantaneous diffusion 15, 16, though additional magnetic field noise in our spectrometer reduces this time to about a millisecond. To circumvent the effect of magnetic field noise, we use the UDD dynamical decoupling sequence [17, with voltage pulses applied between every other pair of decoupling microwave pulses (Fig. 1(a)).

The phase evolution of the electron spin is shown in Fig. 1 (b) as a function of the duration of the voltage pulse for various voltage amplitudes. For unipolar voltage pulses, the echo decays within one period of oscillation (see Supplementary Information) because of the inhomogenous nature of the linear Stark shift component. The application of bipolar voltage pulses can be used to select only the quadratic Stark shift, yielding many phase oscillations, limited only by the effective electric field distribution in the sample. Inhomogeneities in the electric field are expected due to surface roughness and imperfect alignment of the metal plate on the sample, however the Fourier transform of the signal (Fig. 1(c)) shows a Lorentzian distribution which is suggestive of a different mechanism. This could arise from impact ionization events of donor from energetic free electrons under the electric field [18]. Finally, the Stark shift parameters for ${ }^{28} \mathrm{Si}:{ }^{121} \mathrm{Sb}$ where measured to be $\eta_{\mathrm{A}}=-3.54 \pm 0.11 \times 10^{-3} \mu \mathrm{m}^{2} / \mathrm{V}^{2}$ and $\eta_{\mathrm{ge}}=5.3 \pm 1.9 \times 10^{-6} \mu \mathrm{m}^{2} / \mathrm{V}^{2}$, in good agreement with published values 10] (see Supplementary Information).

We now move on to examine the effect of the electric field on the Sb nuclear spin, beginning with an analogous experiments to that above to create a nuclear phase gate. Measurements of the Sb nuclear spin are realised using a Davies ENDOR sequence (Fig. 2(a)) which projects the nuclear spin population onto the electron spin [19, 20]. Figure 2(a-c) illustrates how combining the RF pulses with electric field control enables arbitrary $\mathrm{X}$ and $\mathrm{Z}$ rotations to be performed on the nuclear spin. This demonstrates that for specific values of the voltage pulse duration and amplitude, it is possible to enable or disable the effect of the applied RF pulses (Fig. 2(d)): when the voltage-induced nuclear spin phase shift is equal to $\pi$, the total sequence is always equivalent to a $\pi$ RF pulse, independent of the RF duration $\tau_{R F}$. The conditional operation on the nuclear spin had a total duration of about $0.5 \mathrm{~ms}$ which remains quite long even compared to the nuclear spin coherence times. However, the applied electric field of $0.09 \mathrm{~V} / \mu \mathrm{m}$ is still far from the ionization energy of $\gtrsim 1 \mathrm{~V} / \mu \mathrm{m}$ for donors in silicon [7, 21, so in principle the voltage pulse durations could be significantly reduced.

We next use the electric field to tune the nuclear spin 
(a)

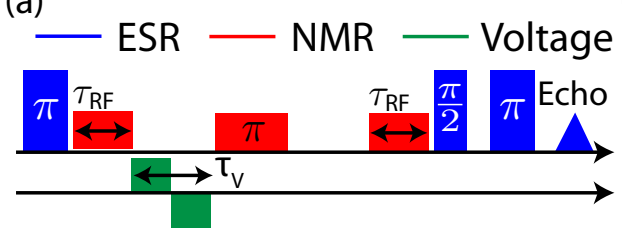

(c)

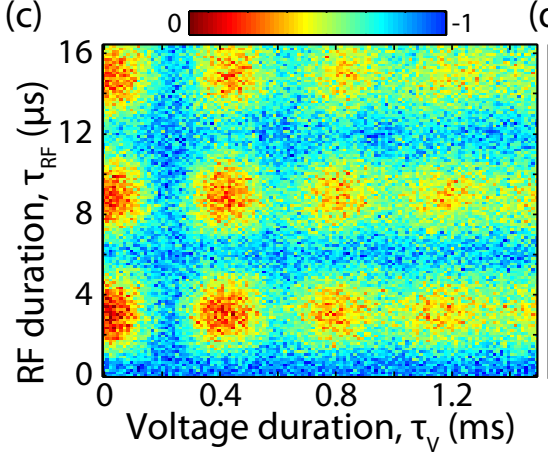

(d) (b)

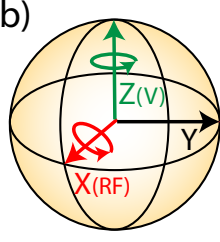

- Voltage ON

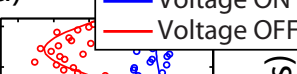

(c)

(a)

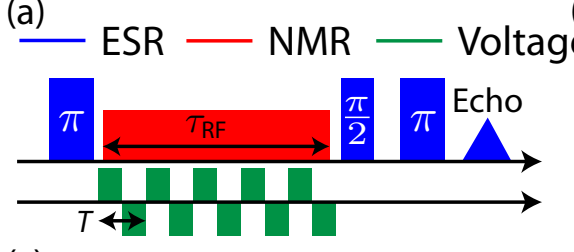

(b)
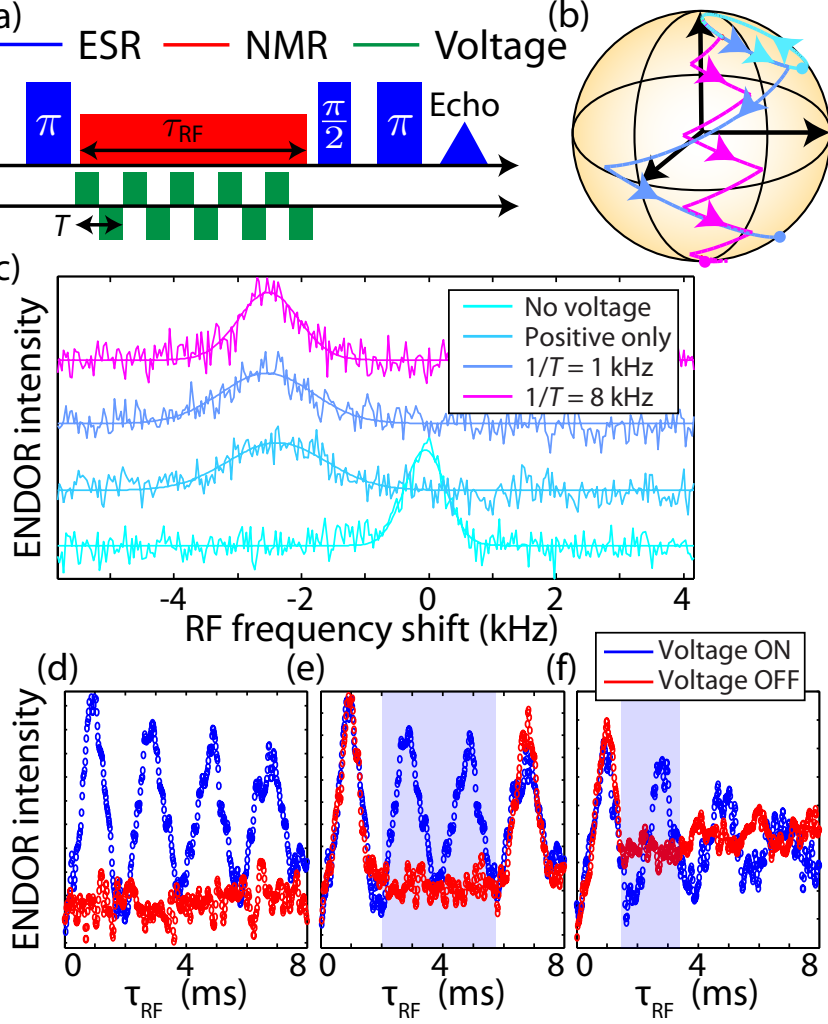

FIG. 3. Electrically-tuning the nuclear spin transition frequency. (a) Davies ENDOR sequence with square wave voltage pulses. (b) Bloch sphere representation of the nuclear spin evolution in the rotating frame at the RF frequency which is resonant under a pure quadratic Stark shift. The linear Stark shift component adds additional off-resonant evolution, which can be compensated by applying a square-wave voltage pulse. (c) ENDOR spectra measured with and without $150 \mathrm{~V}$ pulses, where $\tau_{\mathrm{RF}}=1 \mathrm{~ms}$ corresponding to a $\pi$ pulse. For a single unipolar voltage pulse, the shifted line is broadened by the linear Stark shift. The shifted ENDOR line can be narrowed by increasing the frequency of a square wave bipolar voltage pulse, up to a point, when it becomes limited by electric field inhomogeneity. (d-f) Nuclear spin Rabi oscillations, where: (d) the voltage is constantly applied; (e) the voltage is applied only within the light blue window which starts when the nuclear spin is in an eigenstate; (f) the voltage is applied within the light blue window which starts when the nuclear spin is in a coherent superposition state.

rom the metal plates).

Figure 3(c) shows the ENDOR spectrum around the $m_{\mathrm{S}}=+1 / 2$ nuclear spin transition, recorded for different voltage pulses. Applying a unipolar voltage pulse for the full duration of the RF pulse is sufficient to fully shift the NMR line by more than a linewidth, however, the linewidth broadens due to electric field inhomogeneity and, in particular, the linear Stark effect. The latter can be suppressed using a voltage pulse of alternating polarity (i.e. a square wave with frequency of $8 \mathrm{kHz}$ ) as shown in figure 3(a,b), allowing the Stark-shifted peak to narrow to a similar linewidth to the unshifted peak (see Supplementary Information). The electric field inhomogeneity is not corrected with this sequence and as a result the peak linewidth and intensity cannot be fully recovered.

The ability to tune Sb nuclear spins in and out of resonance with an applied RF field is shown in Fig. 3(d-f), where coherent rotation of the nuclear spins can be completely suppressed, or paused, for some given duration. This works effectively if the voltage pulse is applied when the nuclear spin is in an eigenstate, however, when the nuclear spin is in a coherent superposition, rapid dephasing caused by the applied electric field leads to incomplete recovery of the nuclear spin state after the end of the voltage pulse.

To solve this problem, a hard $\pi$ refocusing RF pulse can 


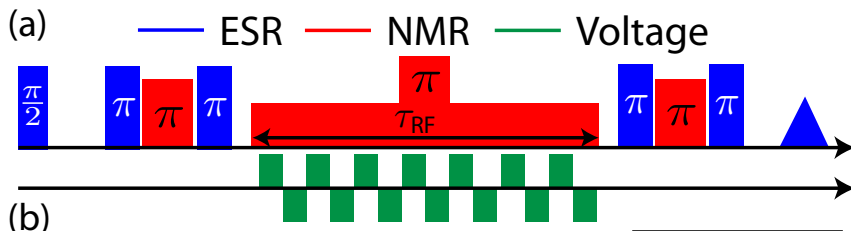

(b)

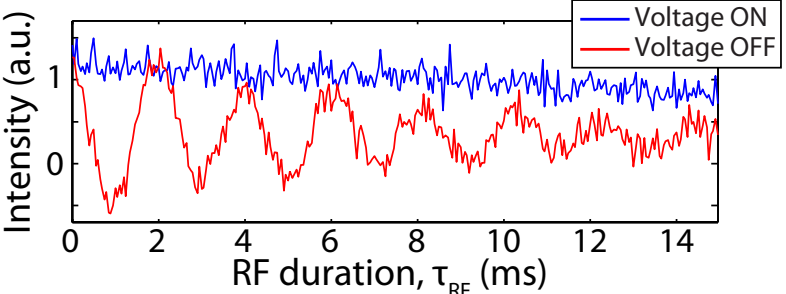

(c)
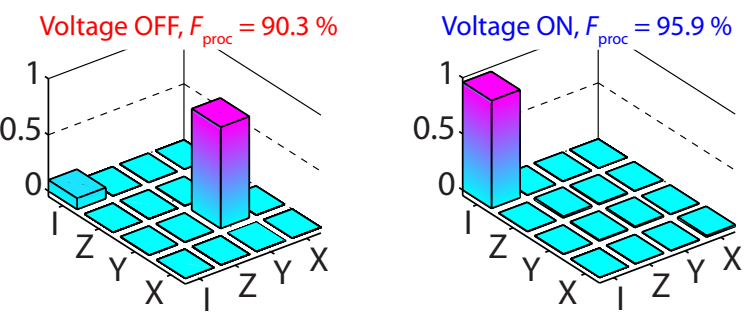

FIG. 4. (a) Sequence taken from 25 used for transferring an electron state to a nuclear state. This is used to produce any input state ( $\pm X, \pm Y, \pm Z$ and identity) due to limited $\mathrm{RF}$ phase control in our experiment. The $\pi \mathrm{RF}$ gate in the middle is large bandwidth $(\approx 30 \mathrm{kHz})$ and refocuses any dephasing when the voltage is on. (b) Rabi oscillations with and without voltages for an input coherent superposition. The oscillations decay quite fast for negative amplitudes as they correspond to even number of $\pi$ pulses which prevent refocusing of the nuclear coherence. (c) Quantum process tomography $\chi_{\text {exp }}$ matrices with voltage on (fidelity compared to a Y rotation) and voltage off (fidelity compared to the identity matrix). Only the real part is shown as the imaginary part is negligible.

be applied half-way through the RF rotation (Fig. 4(a)). It has the same frequency as the other RF pulses but with a much higher bandwidth (shorter, higher power pulse) that excites the nuclear spin regardless of any Stark-shift detuning. As shown in Fig. 4(b), the recovered nuclear spin coherence now decays much more slowly.

With most of these source of errors suppressed, we finally evaluate the performance of the conditional nuclear spin gate using quantum process tomography $[22,25]$ (see Supplementary Information). In the absence of an applied voltage pulse, the gate indeed acts as a $\pi_{Y}$ rotation with a process fidelity $F_{\text {proc }}=\operatorname{Tr}\left(\chi_{\exp } \chi_{\text {ideal }}\right)=90.3 \%$, where $\chi_{\exp }$ and $\chi_{\text {ideal }}$ are the measured and expected process matrices, respectively (Fig. 4(c)). The fidelity is limited here by the inhomogeneous broadening of the nuclear spin, $T_{2 \mathrm{n}}^{*}$. Under an applied voltage pulse, the total gate resembles the identity operation with a fidelity $F_{\text {proc }}=95.9 \%$. Strategies for further increasing the gate fidelities and overcoming effects such as electric field inhomogeneities, including exploring composite pulses to address systematic errors [26, or use of adia- batic sweeps to tune the spins through resonance during the microwave pulse [27, 28. These techniques could be rather insensitive to variations in the electric field and microwave, though at the expense of longer gate durations.

In conclusion, we have shown how the nuclear spin of a donor can be effectively controlled through a combination of RF excitation and an external electric field, either through electrically-controlled phase gates or by detuning spins off resonance. The techniques we discuss for dealing with electric field inhomogeneities could be particularly useful when using electric fields to address a subset of spins in a device, such as tuning out a row of spins in an array. By increasing the electric field closer to onset of donor ionization, these conditional nuclear spin gates could have timescales of order $10 \mu \mathrm{s}$, which compares favourable to the minutes-long nuclear coherence times.

We thank C.C. Lo, A.M. Tyryshkin and S.A. Lyon for valuable discussions. This research is supported by the EPSRC through the Materials World Network (EP/I035536/1) and a DTA, as well as by the European Research Council under the European Community's Seventh Framework Programme (FP7/2007-2013) / ERC grant agreement no. 279781. J.J.L.M. is supported by the Royal Society. The ${ }^{28} \mathrm{Si}$-enriched samples used in this study were prepared from Avo28 material produced by the International Avogadro Coordination (IAC) Project (2004-2011) in cooperation among the BIPM, the INRIM (Italy), the IRMM (EU), the NMIA (Australia), the NMIJ (Japan), the NPL (UK), and the PTB (Germany).

* gary.wolfowicz@materials.ox.ac.uk $^{\text {jjl.morton@ucl.ac.uk }}$

[1] S. Simmons, R. M. Brown, H. Riemann, N. V. Abrosimov, P. Becker, H.-J. Pohl, M. L. W. Thewalt, K. Itoh, and J. Morton, Nature 470, 69 (2011).

[2] J. J. Pla, K. Y. Tan, J. P. Dehollain, W. H. Lim, J. J. L. Morton, D. N. Jamieson, A. S. Dzurak, and A. Morello, Nature 489, 541 (2012)

[3] B. E. Kane, Nature 393, 133 (1998)

[4] C. Hill, L. Hollenberg, A. Fowler, C. Wellard, A. Greentree, and H.-S. Goan, Phys. Rev. B 72, 045350 (2005)

[5] L. Hollenberg, A. Greentree, A. Fowler, and C. Wellard, Phys. Rev. B 74, 045311 (2006)

[6] G. Smit, S. Rogge, J. Caro, and T. Klapwijk, Phys. Rev. B 70, 035206 (2004)

[7] M. Friesen, Phys. Rev. Lett. 94, 186403 (2005)

[8] R. Rahman, C. Wellard, F. Bradbury, M. Prada, J. Cole, G. Klimeck, and L. Hollenberg, Phys. Rev. Lett. 99, 036403 (2007)

[9] R. Rahman, S. Park, T. Boykin, G. Klimeck, S. Rogge, and L. Hollenberg, Phys. Rev. B 80, 155301 (2009).

[10] F. Bradbury, A. M. Tyryshkin, G. Sabouret, J. Bokor, T. Schenkel, and S. A. Lyon, Phys. Rev. Lett. 97, 176404 (2006). 
[11] L. Dreher, T. A. Hilker, A. Brandlmaier, S. T. B. Goennenwein, H. Huebl, M. Stutzmann, and M. S. Brandt, Phys. Rev. Lett. 106, 037601 (2011).

[12] C. C. Lo, S. Simmons, R. L. Nardo, C. D. Weis, A. M. Tyryshkin, J. Meijer, D. Rogalla, S. A. Lyon, J. Bokor, T. Schenkel, and J. J. L. Morton, arXiv Prepr. , 5 (2014), arXiv:1401.6885.

[13] M. Steger, K. Saeedi, M. L. W. Thewalt, J. J. L. Morton, H. Riemann, N. V. Abrosimov, P. Becker, and H.-J. Pohl, Science 336, 1280 (2012)

[14] J. T. Muhonen, J. P. Dehollain, A. Laucht, F. E. Hudson, T. Sekiguchi, K. M. Itoh, D. N. Jamieson, J. C. McCallum, A. S. Dzurak, and A. Morello, arXiv Prepr. , 1 (2014), arXiv:1402.7140

[15] A. Schweiger and G. Jeschke, Principles of pulse electron paramagnetic resonance (Oxford University Press, Oxford, 2001).

[16] A. M. Tyryshkin, S. A. Lyon, A. Astashkin, and A. Raitsimring, Phys. Rev. B 68, 193207 (2003).

[17] G. Uhrig, Phys. Rev. Lett. 98, 100504 (2007)

[18] W. Kaiser and G. Wheatley, Phys. Rev. Lett. 3, 334 (1959)

[19] E. R. Davies, Phys. Lett. A 47, 1 (1974)

[20] A. M. Tyryshkin, J. J. L. Morton, A. Ardavan, and S. A. Lyon, J. Chem. Phys. 124, 234508 (2006)

[21] S. Žurauskas and A. Dargys, Phys. status solidi 121, 385 (1984)

[22] A. Childs, I. Chuang, and D. Leung, Phys. Rev. A 64, $012314(2001)$

[23] M. Ježek, J. Fiurášek, and Z. Hradil, Phys. Rev. A 68, $012305(2003)$

[24] J. O'Brien, G. Pryde, A. Gilchrist, D. James, N. Langford, T. Ralph, and A. White, Phys. Rev. Lett. 93, $080502(2004)$

[25] J. J. L. Morton, A. M. Tyryshkin, R. M. Brown, S. Shankar, B. W. Lovett, A. Ardavan, T. Schenkel, E. E. Haller, J. W. Ager, and S. A. Lyon, Nature 455, 1085 (2008)

[26] S. Wimperis, J. Magn. Reson. Ser. A 109, 221 (1994)

[27] A. Tannús and M. Garwood, NMR Biomed. 434, 423 (1997)

[28] H. Wu, E. M. Gauger, R. E. George, M. Möttönen, H. Riemann, N. V. Abrosimov, P. Becker, H.-J. Pohl, K. M. Itoh, M. L. W. Thewalt, and J. J. L. Morton, Phys. Rev. A 87, 032326 (2013) 


\section{Supplementary information for "Conditional control of donor nuclear spins in silicon using Stark shifts"}

\section{STARK SHIFT IN ANTIMONY DONORS}

In order to estimate $\eta_{\mathrm{A}}$ (hyperfine shift) and $\eta_{\mathrm{g}_{\mathrm{e}}}$ (electron g-factor shift), the frequency shift induced by the Stark effect is measured using the sequence in Fig. 1(a) of the main text. As both parameters are quadratic in the electric field, their values cannot be distinguished using a single ESR transition. However, by taking advantage of the varying values of $d f / d A$ and $d f / d g_{\mathrm{e}}$ for the different ESR transitions, it is possible to estimate the two parameters. In particular, $d f / d A \approx m_{\mathrm{I}}=-5 / 2 \ldots 5 / 2$ for magnetic fields between $310 \mathrm{mT}$ and $350 \mathrm{mT}$ at 9.7 $\mathrm{GHz}$, and so varies quite strongly.

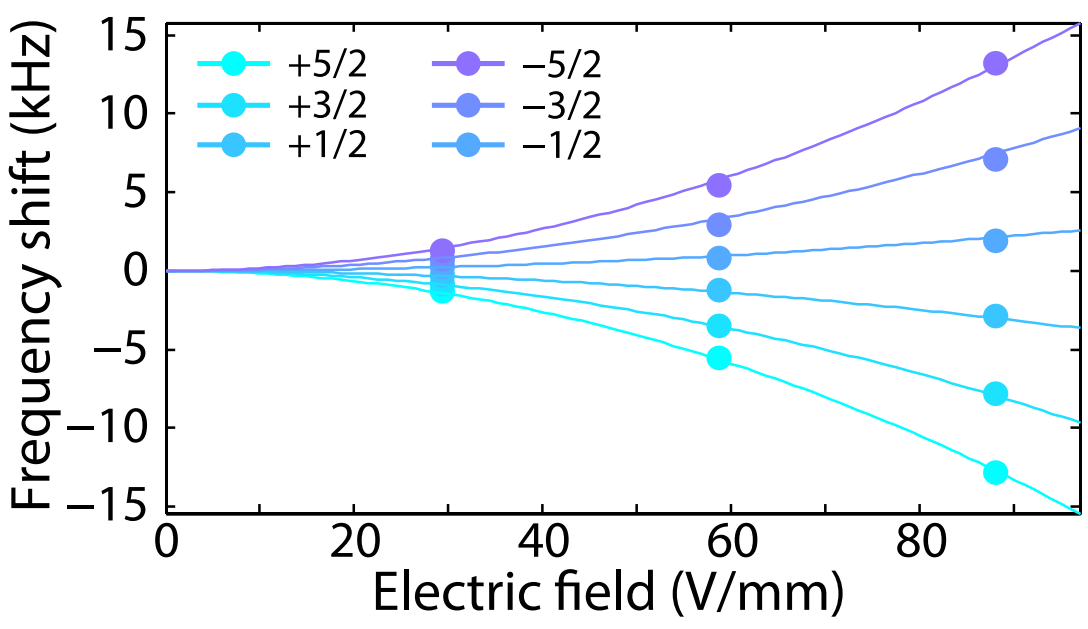

FIG. 1. Stark shift as a function of voltage for each ESR transition in ${ }^{121} \mathrm{Sb}$. The filled circles are the experimental values while the lines are given by the fitted model (Eq. 1 in the main text). Error bars are smaller than the size of the circles.

The measured shifts for each voltage and each $m_{\mathrm{I}}$ state are simultaneously fitted to the only two free parameters: $\eta_{\mathrm{A}}$ and $\eta_{\mathrm{g}_{\mathrm{e}}}$. There are two sources of error: fitting errors and the uncertainty in the electric field. In the setup, the voltage pulses with amplitude up to $1.2 \mathrm{~V}$ are produced by a pulse generator, and then amplified by an operational amplifier with gain $G=126 \pm 1$. The overall circuit has an $\mathrm{RC}$ constant $\tau_{\mathrm{RC}}=6 \pm 0.6 \mu$ s which is also taken 
into account in the measurement sequence. The phase shift accumulated by the spins from a voltage pulse can be written as:

$$
\Delta \phi=2 \pi \alpha\left(\eta_{\mathrm{ge}_{\mathrm{e}}}, \eta_{\mathrm{A}}\right)\left[\int_{0}^{\tau_{\mathrm{V}}} E_{0}^{2}\left(1-e^{-t / \tau_{\mathrm{RC}}}\right)^{2} d t+\int_{0}^{+\infty} E_{0}^{2}\left(1-e^{-\tau_{\mathrm{V}} / \tau_{\mathrm{RC}}}\right)^{2}\left(e^{-t / \tau_{\mathrm{RC}}}\right)^{2} d t\right]
$$

where $\tau_{\mathrm{V}}$ is the voltage duration, $E_{0}$ is the maximum voltage amplitude and $\alpha\left(\eta_{\mathrm{ge}_{\mathrm{e}}}, \eta_{\mathrm{A}}\right)=$ $\frac{d f}{d A} \eta_{A} A+\frac{d f}{d g_{\mathrm{e}}} \eta_{\mathrm{g}_{\mathrm{e}}} g_{\mathrm{e}}$. If the Stark shift response was linear, the integral would just be equal to $E_{0} \tau_{\mathrm{V}}$ and there would be no error from the rise and set time of the pulse. However in this case, the integral for the quadratic response is equal to $E_{0}^{2}\left(\tau_{\mathrm{V}}-\tau_{\mathrm{RC}}\left(1-e^{-\tau_{\mathrm{V}} / \tau_{\mathrm{RC}}}\right)\right)$ giving a non-negligible error from the $\mathrm{RC}$ constant. For the estimation of $\eta_{\mathrm{A}}$ and $\eta_{\mathrm{g}_{\mathrm{e}}}$, this error is corrected by renormalizing $\tau_{\mathrm{V}}$ such that:

$$
\tau_{\mathrm{V}}^{\prime}=\tau_{\mathrm{V}}-2 N \tau_{\mathrm{RC}}\left(1-e^{-\tau_{\mathrm{V}} /\left(2 N \tau_{\mathrm{RC}}\right)}\right)
$$

where $N=2$ is the number of bipolar pulses in UDD4 (the factor 2 in front of $N$ accounts for both positive and negative pulses).

\section{UNIPOLAR AND BIPOLAR SEQUENCES}

In principle, the Stark shift should be purely quadratic in electric field. However, there is a component of the Stark shift which is linear in electric field for donors in materials with strain and internal electric fields. Unipolar voltage pulses produce frequency shifts arising from both the linear and quadratic terms, however for a bipolar pulse, the linear term cancels [1]. As the linear contribution originates from inhomogeneous environments across the ensemble, it results in a decay of the electron spin echo as shown in Fig. 2. For a unipolar pulse, the oscillations have a decay times of $60 \mu \mathrm{s}$, about 3 times faster than for the bipolar one.

\section{ELECTRICALLY-CONTROLLED PHASE GATE}

The electrically-controlled phase gate presented in Fig. 2 of the main text combines arbitrary $\mathrm{X}$ and $\mathrm{Z}$ rotations. The observed dynamics can be simulated using the following 


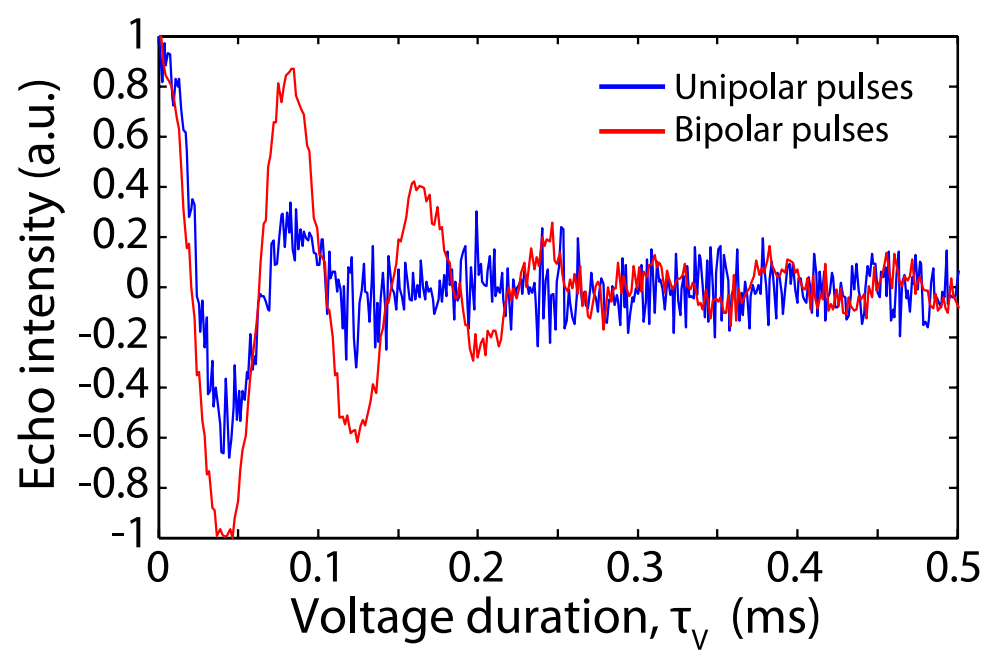

FIG. 2. Phase evolution in the electron spin echo as a function of the voltage pulse duration, for both unipolar and bipolar voltage pulses, measured by observing the in-phase component of the echo in quadrature detection. The decay in the case of the unipolar pulses is due to the inherently inhomogeneous linear Stark shift term, which is canceled out in the bipolar case.

equation:

$$
S=\frac{1}{2}\left[\left(\cos \left(\theta_{X}\right)^{2}-\sin \left(\theta_{X}\right)^{2} \cos \left(\theta_{Z}\right) D\left(\theta_{Z}\right)\right) D\left(\theta_{X}\right)-1\right]
$$

where $\theta_{X}\left(\tau_{\mathrm{RF}}\right)$ and $\theta_{\mathrm{Z}}\left(\tau_{\mathrm{V}}\right)$ are respectively the rotation angles of the $\mathrm{X}$ and $\mathrm{Z}$ gates, and $D\left(\theta_{\mathrm{X}}\right)$ and $D\left(\theta_{\mathrm{Z}}\right)$ two phenomenological distributions for the electric field and the AC magnetic field across the ensemble, leading to finite rotation fidelities. The simulation is compared to the experiment in Fig. 3. For $D\left(\theta_{\mathrm{Z}}\right)$, we used a Lorentzian distribution, as measured in a Ramsey interference experiment (Fig. 1 of main text). For $D\left(\theta_{\mathbf{X}}\right)$, nuclear Rabi oscillations in the absence of any voltage show a Gaussian decay, as is commonly observed.

\section{SQUARE WAVE PULSES AND LINEAR SHIFT SUPPRESSION}

The linear Stark shift can be readily canceled by the application of bipolar pulses in the Ramsey interference experiment. However, when simply detuning the spins (Fig. 4), the Stark shift will interfere directly with the precession from an external RF excitation.

The interaction Hamiltonian due to the application of a resonant RF (magnetic field) and a (quasi-DC) electric field pulse is, in the rotating frame:

$$
H_{ \pm}=\Omega_{\mathrm{R}} S_{\mathrm{X}} \pm \delta \omega(E) S_{\mathrm{Z}}
$$


(a)

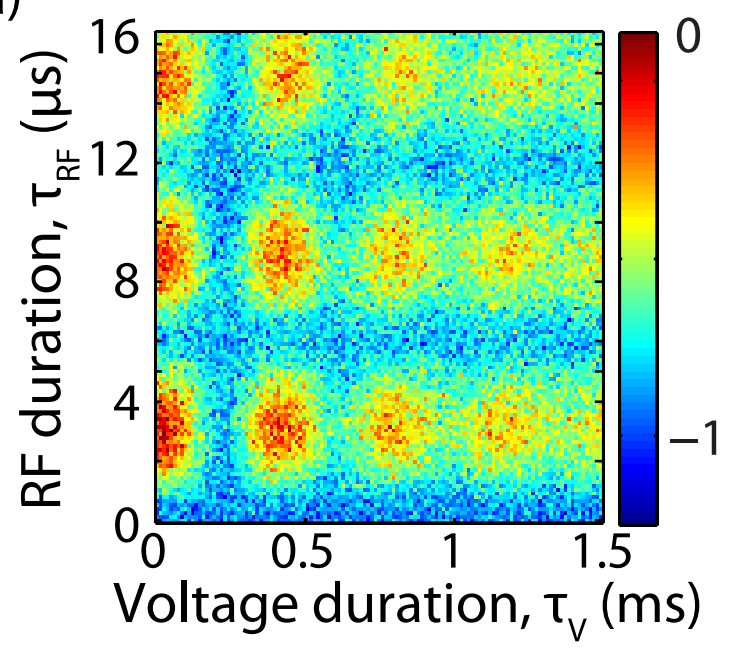

(b)

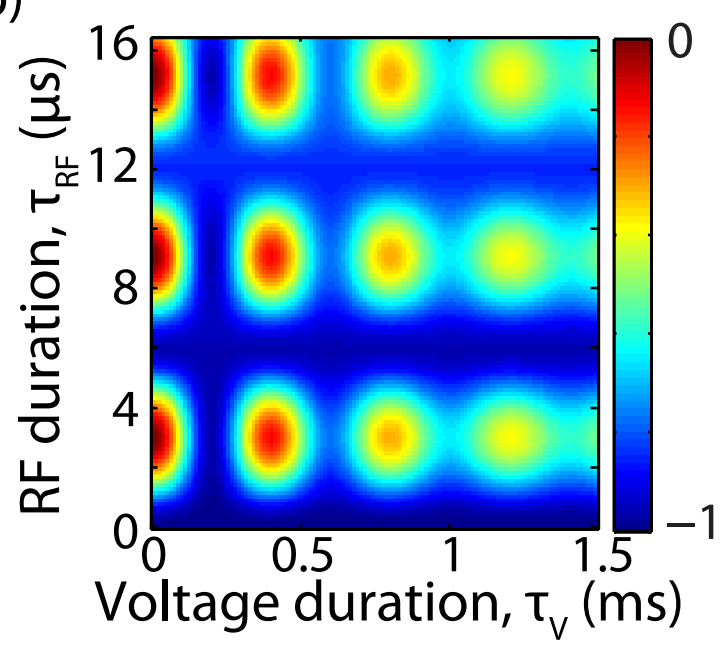

FIG. 3. $\mathrm{X}$ and $\mathrm{Z}$ rotations using RF and voltage pulses. (a) Experiment (reproduced from main text). (b) Simulation using Eq. 2. The Rabi frequencies for the $\mathrm{X}$ and $\mathrm{Z}$ rotations are $80 \mathrm{kHz}$ (the measured oscillation are 2 times faster due to sweeping $2 \tau_{\mathrm{RF}}$ in the sequence) and $2.5 \mathrm{kHz}$, respectively. The linewidths for the $D\left(\theta_{\mathrm{X}}\right)$ and $D\left(\theta_{\mathrm{Z}}\right)$ distributions are $50 \mathrm{kHz}$ and $1.5 \mathrm{kHz}$, respectively.

where $\Omega_{\mathrm{R}}$ is the RF Rabi frequency, $\delta \omega(E)$ the electric field-induced shift and $S_{\mathrm{X}, \mathrm{Z}}$ the Pauli matrices for spin $1 / 2$. The voltage polarity is flipped with a period $T=T_{\text {end }} / N$, where $T_{\text {end }}$ is the total evolution time and $N$ is the period number, leading to the following propagator $U$ :

$$
U=\left(e^{-i H_{+} \frac{T_{\text {end }}}{2 N}} e^{-i H_{-}-\frac{T_{\text {end }}}{2 N}}\right)^{N}
$$

According to the Suzuki-Trotter expansion for the matrix exponential [2], the propagator becomes for large $N$ :

$$
U^{N \rightarrow+\infty} \rightarrow e^{-i\left(H_{+}+H_{-}\right) T_{\text {end }} / 2}=e^{-i \Omega_{\mathrm{R}} S T_{\mathrm{X}} T_{\text {end }}}
$$

In this case, the contribution from the detuning $\delta \omega(E)$ cancels out, and the effect of the linear Stark shift on the linewidth can thus be suppressed. This approximation remains valid as long as the frequency $1 / T$ is faster than the evolution rate $\sqrt{\Omega_{\mathrm{R}}^{2}+\delta \omega^{2}(E)} /(2 \pi)=2.5 \mathrm{kHz}$. This is simulated in figure $4(\mathrm{c})$. 
(a)

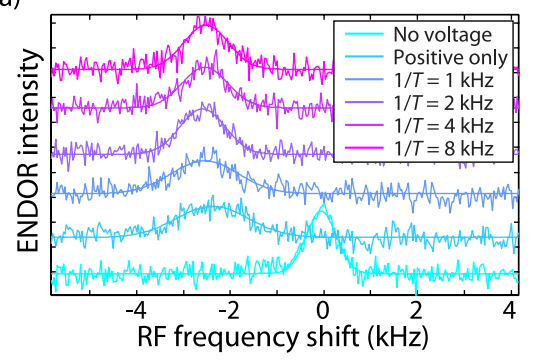

(b)

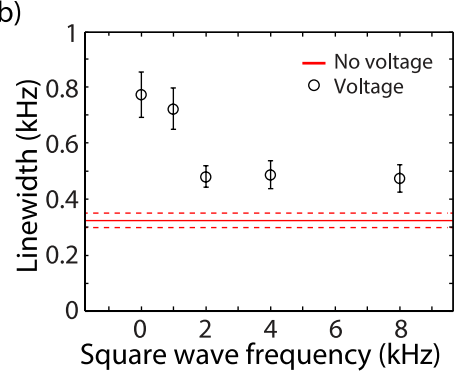

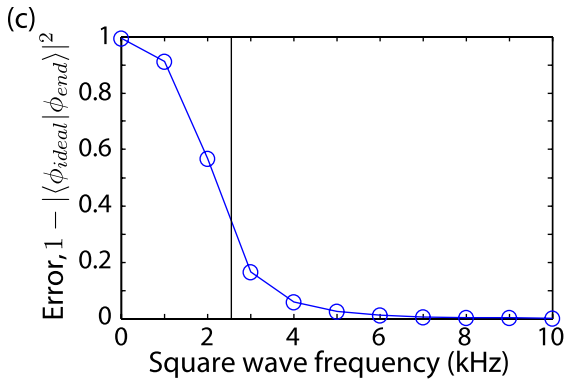

FIG. 4. (a) ENDOR spectra, in the absence of a voltage pulse, under a unipolar voltage pulse, and under a bipolar square-wave voltage pulse of various frequencies. (b) Fitted ENDOR linewidth as a function of the voltage square wave frequency. The linewidth can be partially recovered with higher frequencies, but is eventually limited by electric field inhomogeneity. The dashed lines are the error bars for the "no voltage pulse" case. (c) Simplified simulation of the experiment, considering a simple spin-1/2 with only a linear Stark effect contribution, under square wave pulses of varying frequency. Starting from an initial state $|\phi\rangle=\left[\begin{array}{ll}1 & 0\end{array}\right]^{\mathrm{T}}$, the system evolves to $\left|\phi_{\text {end }}\right\rangle$ under an RF excitation. In the absence of the linear Stark shift, the spin performs a $\pi$-rotation to $\left|\phi_{\text {ideal }}\right\rangle=\left[\begin{array}{ll}0 & 1\end{array}\right]^{\mathrm{T}}$. In the presence of the shift, the application of a square wave slowly cancels it as the frequency becomes larger than the evolution rate $\sqrt{\Omega_{\mathrm{R}}^{2}+\delta \omega^{2}(E)}$ (black line).

\section{PROCESS TOMOGRAPHY}

The application of an electric field allowed us to pause the Rabi oscillations, or, more generally, disable the quantum operation performed via resonant RF excitation of the nuclear spin. The fidelity of this conditional operation can be measured by performing quantum process tomography (QPT) and comparing the measured operation with the ideal one, which is either the $\pi$-rotation with voltage or the identity without (i.e. the RF pulse has no effect as the spins are off-resonance from the voltage). QPT aims at finding the process that transfers any input states to specific output states [3]. This is realized by reconstructing from experimental measurements a completely positive map $\epsilon$ of this transformation, represented by the matrix $\chi$, such that for an input density matrix $\rho$, the output is:

$$
\epsilon(\rho)=\sum_{\mathrm{m}, \mathrm{n}=0}^{\mathrm{d}^{2}-1} \chi_{\mathrm{mn}} A_{\mathrm{m}} \rho A_{\mathrm{n}}^{\dagger}
$$

where $\left(A_{\mathrm{m}}\right)$ is the Pauli basis $\left\{\mathbb{1}, \sigma_{\mathrm{X}}, \sigma_{\mathrm{Y}}, \sigma_{\mathrm{Z}}\right\}$ and $d=2$ the dimension of the Hilbert space. 
In order to create $\chi$, we first need to construct the 'null' and 'evolved' density matrices $\rho_{\mathrm{i}}$ and $\rho_{\mathrm{f}}$ from the experiments using quantum state tomography, which can be done for mixed (ensemble) states using the method given in the supplementary information of Ref [4]. The inputs $\left\{\mathbb{1}, \pm \sigma_{\mathrm{X}}, \pm \sigma_{\mathrm{Y}}, \pm \sigma_{\mathrm{Z}}\right\}$ are transformed by the combined sequence of $\mathrm{RF}$ and voltage pulses and then measured in the $\left\{\sigma_{\mathrm{X}}, \sigma_{\mathrm{Y}}, \sigma_{\mathrm{Z}}\right\}$ basis. To minimise the effect of state preparation and measurement errors, the 'null' state is that measured at the end of the sequence (Fig. 4(a) in main text) in the absence of RF or voltage pulses. The two 'evolved' state density matrices are those obtained in the presence of RF rotation excitation and with/without voltage pulses. In order to obtain pseudo-pure matrices, the norm of the signal (in the measurement basis) from the 'null' state is used to normalize all the density matrices. The reconstructed matrices are shown in Fig. 5 for the case where voltage pulses are "ON". In the ideal case, for the voltage pulses on the nuclear spins should be detuned from the RF pulse and $\rho_{\mathrm{f}}=\rho_{\mathrm{i}}$. We can compute the state fidelity of $\rho_{\mathrm{f}}$ onto $\rho_{\mathrm{i}}: F=\operatorname{Tr}\left(\sqrt{\rho_{\mathrm{f}}} \rho_{\mathrm{i}} \sqrt{\rho_{\mathrm{f}}}\right)^{2}$, which gives in average for all input states $\bar{F}=0.97$.
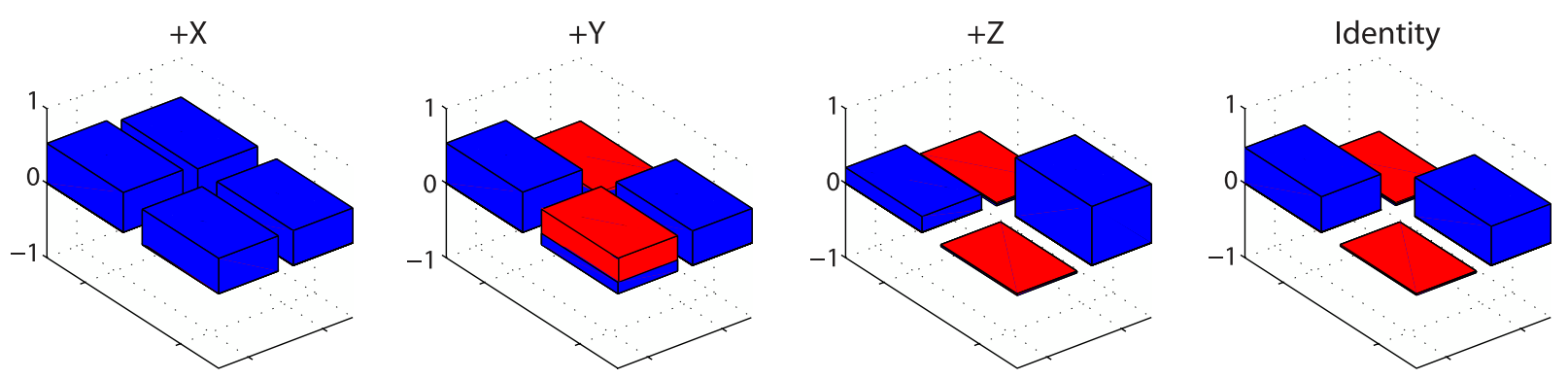

FIG. 5. Quantum state tomography: density matrix reconstruction from echo intensities of the experiment in Fig. 4 of the main text with voltage "ON". (Blue) real and (red) imaginary part of the density matrix.

The process matrix $\chi$ can be constructed by simple linear inversion [5], however the resulting matrix is usually not physical, i.e. not Hermitian positive. The solution is then to find out the closest physical operation via least square fitting of the $\rho_{\mathrm{i}}$ and $\rho_{\mathrm{f}}$ matrices, as described in [3]. The $\chi$ matrices presented in Fig. 4(c) of the main text are calculated with this method. 
[1] F. Bradbury, A. M. Tyryshkin, G. Sabouret, J. Bokor, T. Schenkel, and S. A. Lyon, Phys. Rev. Lett. 97, 176404 (2006).

[2] N. Hatano and M. Suzuki, in Quantum Annealing Other Optim. Methods, Vol. 68 (Springer, 2005) pp. 37-68.

[3] J. O'Brien, G. Pryde, A. Gilchrist, D. James, N. Langford, T. Ralph, and A. White, Phys. Rev. Lett. 93, 080502 (2004).

[4] J. J. L. Morton, A. M. Tyryshkin, R. M. Brown, S. Shankar, B. W. Lovett, A. Ardavan, T. Schenkel, E. E. Haller, J. W. Ager, and S. A. Lyon, Nature 455, 1085 (2008).

[5] A. Childs, I. Chuang, and D. Leung, Phys. Rev. A 64, 012314 (2001). 